\title{
Technical Note: Why Is Vibration an Issue for Dissolution Testing?
}

\author{
John Burmicz \\ Pharma Test GmbH, Siemensstrasse 5 \\ D-63512, Hainburg. Germany
}

e-mail:drjohn.burmicz@pharma-test.de

\section{INTRODUCTION}

t has become apparent, in the recent past, that vibra-

tion within the tablet dissolution testing environment is

a real issue.

Although this may not have been too apparent in normal routine dissolution testing, the real impact of vibration has become a focal point as a result of the Prednisone "Calibrator Tablet" tests, which generally have to be performed every six months on each instrument within the testing environment. Prednisone tablets are referred to as "disintegrating" tablets because they break down in the medium very quickly to form a powdered mass in the basket (Apparatus 1) or a fairly well-defined cone under the Paddle (Apparatus 2). The second system validation test is made using Salicylic Acid tablets, which are "non-disintegrating," so they keep their form throughout the test and slowly dissolve.

The Prednisone tablet is $10 \mathrm{mg}$ in active content, and so it has a large percentage of excipients present; in contrast, the Salicylic Acid tablet is made entirely of salicylic acid with no excipients present.

\section{WHAT CAN GO WRONG?}

The discussion will be limited to the Prednisone tablets since their dissolution rate is more affected by the vibration issues under discussion. However, the influences of the sources outlined below can have an effect on all types of dissolution tests, and their influences cannot always be absolutely defined. Their elimination or minimization, however, will lead to a corresponding reduction in systematic errors, which is to be desired.

Up to this point, there were certain "anomalies" noted with Prednisone tests, and many of the more spurious results of these tests were attributed to various causes, normally the quality of the tablets themselves. The issue of test reliability appeared to become a particular problem when the formulation was changed from a 50-mg tablet to a 10-mg tablet. Up to this point, there were no particular influences noted with the 50 -mg formulation. The 10-mg formulation, however, presented some challenges to the operator and his/her working environment, and it became apparent that the dissolution release rate was greatly influenced by two main factors, dissolved gasses and vibration.

On many occasions, the combination of poor deaeration and vibration can work together to produce all manner of strange effects. The dissolved gasses issue can be easily resolved using a suitable deaeration technique. At this point, it is reasonable to draw a difference between "degassing" and "deaeration." Degassing is a technique whereby dissolved gasses with a high solubility product can be displaced by a gas with a lower solubility product. Typically, helium can be used to displace both nitrogen and oxygen in aqueous media. Deaeration, on the other hand, is a method by which we physically remove the dissolved gasses from the medium. If we follow the USP requirements, this can be achieved by a combination of heating, vacuum, and circulation (1). Medium deaeration today is a well-defined subject and should not be an issue.

\section{WHAT ABOUT VIBRATION?}

Vibration sources can be very diverse in origin. Some of the sources are internal sources, external sources, and intermittent and operational sources.

\section{Internal Sources}

\section{The Heater/Circulator}

These basically arise from the instrument construction and the types of components used to manufacture the machine. The first major source is that of the external heater/circulator. These devices are normally of a basic design with a reasonably powered pump that takes water from the bath, circulates it through a heater element, and then returns it to the bath. Unfortunately, this design may not be vibration-proof. These devices may generate a lot of $50 / 60-\mathrm{Hz}$ noise, which is transmitted to the dissolution bath. The construction of the lab bench may also prove to be an amplifier for this "transmission."

\section{The Drive Motor}

The next source is that of the tool rotational motor, drive belts, and bearings. The rotational motor can be a source of vibration. It normally is a DC motor with a gearbox attached. The gearbox has to be maintained and checked for squeaks and rattles.

\section{The Drive Belts}

The drive belts fall into two classes, those that are expected to drive all tool shafts from a single belt and those that have a high contact angle with the drive shaft mechanism (so multiple belt drives). Generally, the quality of the belt will play a big role in the generation of higher frequency vibration, which is then transmitted down the 
tool shaft adding another enforced dimension to the tablet disintegration and dissolution process. Noise generated in this way will turn the tool shaft into a type of ultrasonic disintegration probe resulting in higher dissolution rates, especially for Apparatus 1 baskets. The effect is not so profound with Apparatus 2 paddles, probably due to the relative dissociation of the tool and the tablet, and the damping effect of the medium. Using Prednisone with Apparatus 1, you can tell if there has been significant high frequency vibration transmission by the amount of "escaped" powder at the base of the dissolution vessel. Inspection of the belts will show immediately if there has been some wear, and if they are shiny, then the metal or plastic drive cog wheels at the top of tool shaft may generate noise. You could either replace the belts or use a silicone grease based PTFE loaded spray (sparingly), which will smooth out the noise. The effect of this type of loaded lubricant can be quite dramatic.

\section{The Bearings}

The bearings themselves can be either lubricated for life or grease-packed (generally in older models). The bearings are not generally checked for wear and tear themselves but are tested as a function of the tool wobble or run-out test, which is part of the OQ procedure. Of course, there is the need to distinguish between tool wobble and bearing-induced wobble. The bearing contribution to the overall wobble can be easily measured at the part of the tool that passes through the bearing and is located at the top of the dissolution tester head. If the tools are in a sealed ("capped") bearing, then this is not possible. Your only guide will then be the wobble or run-out at the end of the tool shaft. However, the bearing/tool wobble may not be totally indicative of bearing noise. Wobble measurements may not reveal sporadic bearing noise (typically a low frequency knocking). This can influence the dissolution rate very significantly, especially with Apparatus 1 baskets. Excessive tool wobble may be indicative of distorted tools or worn bearings. An experienced investigator may be able to feel and pinpoint any unusual bearing behavior.

\section{External Sources}

So this is the mechanical side. But what about the environment directly around the instrument? Almost no attention is paid to environmental sources of vibration. Only on some rare occasion is there such an influence that the lab is more or less forced to concede that there have been hidden forces at work.

A typical example is that of a 12-position bath placed in a laboratory where most of the instrument rested on an old weighing table and the remaining third of the instrument rested on an extension to this weighing table that was made from a "kitchen-style" worktop with a thin metal frame as a support. During the Prednisone tests, it was noted three times that vessels $5,6,11$, and 12 were failing the test, while 1, 2, 3, and 4 in the front row as well as $7,8,9$, and 10 in the back row were well within the range. The problem was a BET Surface Area Analyzer, which used a high-vacuum pump to degas powdered samples prior to analysis. The pump, typically the least serviced part of the instrument system, was running more or less full time and was resting on the floor, but only about $0.5 \mathrm{~m}$ from the base of the frame support. The vibration transmission was easily spotted in the poorly supported dissolution vessels as a series of concentric ring patterns on the surface of the media. With the pump off, all was well.

This was, by no means, the only potential culprit. Old refrigerators with ancient compressors are also a common source of vibration.

\section{Intermittent and Operational Sources}

The trouble with any intermittent problem is that it may not always be there. The best example is the slamming door. Portable A/C units can also be a problem.

Intermittent sources can also be operator-generated. Some instruments have electric-head drives (to raise and lower the head), and some are manually operated, either with a pneumatically assisted swing head or with a guided vertical drop. Both of these mechanisms can be sources of problems if not smoothly placed into the operating position. This, again, is particularly the case with Apparatus 1 baskets. Sudden movements during the disintegration of the Prednisone Tablet can fire a shower of tablet material out of the basket and into the medium. A failed test is not far off.

\section{MODERN DISSOLUTION TESTERS AND VIBRATION}

Modern test instruments constructed with up-to-date materials should be designed with the dissolution user in mind and not just for economical construction. As far as the USP is concerned, it has been noted that there is only a brief mention of vibration requirements consisting of the statement that "no part of the assembly, including the environment in which the assembly is placed, contributes significant motion, agitation, or vibration beyond that due to the smoothly rotating stirring element" (1).

Other publications have also cited potential errors in terms of dissolution testing, and among the biggest influences were vibration and medium deaeration (2).

The effect of vibration on instruments from various suppliers has also been investigated and is documented in existing literature (3). The method of vibration analysis and investigation was also reviewed by this independent body, and the results make quite interesting reading. The main points are:

- The method for measuring and documenting vibration is important.

- Are you measuring vibration in one axis or are some axes being "damped"? 
- It is important to use a real vibration meter that is sensitive to vibration in all axes, rather than one that simply attaches onto the instrument surface.

\section{References}

1. USP Medium preparation advice for Prednisone RS Tablets. USP publication included with each set of Calibrator Tablets.
2. Gray, V. Challenges to the Dissolution Test, including Equipment Calibration. Dissolution Technol. 2006, 13 (2), 6-9.

3. Vangani, S.; Flick, T.; Tamayo, G.; Chiu, R.; Cauchon, N. Vibration Measurements on Dissolution Systems and Effects on Dissolution of Prednisone Tablets RS.

Dissolution Technol. 2007, 14 (1), 6-14. 